\title{
Erratum to: Streptosporangium shengliensis sp. nov., a novel actinomycete isolated from a lake sediment
}

\author{
Xinhui Zhang • Chongxi Liu • Yuejing Zhang • \\ Haiyan Wang · Shurui Wang · Chuang Li • \\ Xiangjing Wang $\cdot$ Wensheng Xiang
}

Published online: 19 December 2013

(C) Springer Science+Business Media Dordrecht 2013

\section{Erratum to: Antonie van Leeuwenhoek DOI 10.1007/s10482-013-0070-7}

Subsequent to the publication of the above paper it has been brought to our attention that the species epithet proposed for the taxon represented by strain NEAU$\mathrm{GH} 7^{\mathrm{T}}$ is not grammatically correct as "Streptosporangium shengliensis" should have been proposed as Streptosporangium shengliense. We here propose the corrected name for the taxon represented by strain NEAU-GH7 $7^{\mathrm{T}}$.

\section{Description of Streptosporangium shengliense sp. nov.}

Streptosporangium shengliense (sheng.li.en'se. N.L. neut. adj. shengliense pertaining to Shengli Park, from where the strain was isolated).

The description of the species is as given for "Streptosporangium shengliensis" in Zhang et al. (2014) Antonie van Leeuwenhoek (DOI 10.1007/s10482-013-0070-7).

The type strain is NEAU-GH7 $7^{\mathrm{T}}\left(=\mathrm{CGMCC} 4.7105^{\mathrm{T}}=\right.$ $\operatorname{DSM} 45881^{\mathrm{T}}$ ).
The online version of the original article can be found under doi:10.1007/s10482-013-0070-7.

X. Zhang $\cdot$ C. Liu $\cdot$ Y. Zhang $\cdot$ S. Wang $\cdot$

C. Li · W. Xiang $(\bowtie)$

School of Life Science, Northeast Agricultural University, No. 59 Mucai Street, Xiangfang District, Harbin 150030, People's Republic of China

e-mail: xiangwensheng@neau.edu.cn

H. Wang $\cdot$ X. Wang $(\bowtie)$

Key Laboratory of Microbial Drug Engineering of Heilongjiang Provincial Education Committee, Harbin 150030, People's Republic of China

e-mail: wangneau2013@163.com 